\title{
Society for Developmental Biology
}

9650 Rockville Pike, Bethesda, MD 20814-3998, USA

DEVELOPMENTAL

BIOLOGY

T: (301) 634-7815 • F: (301) 634-7825

sdb@sdbonline.org•http://www.sdbonline.org

\section{Position Statement from the Society for Developmental Biology on Genomic Editing in Human Embryos}

The Board of Directors of the Society for Developmental Biology (SDB) and the Editors of its official journal Developmental Biology are very concerned about the recently published study applying CRISPR/Cas9 genome editing technology to human embryos (1). SDB supports a voluntary moratorium by members of the scientific community on all manipulation of preimplantation human embryos by genome editing. Such studies raise deep ethical concerns on their own, and in addition could lead to unanticipated consequences if manipulated embryos were implanted into a womb and allowed to develop to term.

Scientists worldwide apply genome editing technologies to a broad range of model organisms and human cells in culture, and these powerful tools hold great promise in helping to advance basic science knowledge and develop therapeutic approaches to human diseases and congenital defects. Some studies using these technologies in non-human embryos have reported unexpected genome disruptions, including gene deletions and creation of unintended mutations. This first published study (1) using CRISPR/Cas9 mediated-editing in human embryos reports even higher rates of unintended mutations than seen in those previous studies. These findings make further attempts to edit human embryos dangerous and unethical.

While the published study utilized human embryos with three nuclei (tripronuclei-3PN) that cannot develop to term in order to minimize ethical concerns, SDB feels that the use of human embryos in genome engineering research opens the door to applications with current risks that are ethically unacceptable. It is important, however, that the public be educated on the positive uses of genome editing technologies in model organisms and in human somatic cells. Such uses hold enormous promise for advancing scientific knowledge. Legitimate concerns about applying genome editing to human embryos should not impede its use in basic science studies.

1. Liang, P. et al. Protein Cell (2015) DOI 10.1007/s13238-015-0153-5 